Annales Geophysicae (2001) 19: 1449-1460 (C) European Geophysical Society 2001

\title{
Cluster observes the Earth's magnetopause: coordinated four-point magnetic field measurements
}

\author{
M. W. Dunlop ${ }^{1}$, A. Balogh ${ }^{1}$, P. Cargill ${ }^{1}$, R. C. Elphic ${ }^{2}$, K.-H. Fornaçon ${ }^{3}$, E. Georgescu ${ }^{4}$, F. Sedgemore-Schulthess ${ }^{5}$, and \\ the FGM team \\ ${ }^{1}$ Space and Atmospheric Physics Group, Imperial College, London SW7 2BZ, UK \\ ${ }^{2}$ Los Alamos National Laboratory, Los Alamos, NM 87545, USA \\ ${ }^{3}$ Institut für Geophysik und Meteorologie, TUB, Braunschweig, Germany \\ ${ }^{4}$ Max-Planck-Institut für extraterrestrische Physik, Garching, Germany \\ ${ }^{5}$ DSRI, Copenhagen, Denmark
}

Received: 21 March 2001 - Revised: 27 June 2001 - Accepted: 11 July 2001

\begin{abstract}
The four-spacecraft Cluster mission has provided high-time resolution measurements of the magnetic field from closely maintained separation distances $(200-600 \mathrm{~km})$. Four-point coverage of the Earth's magnetopause began on the 9 and 10 November 2000 when all spacecraft first exited the dusk-side magnetosphere at about 19:00 LT, providing extensive coverage of the near flank magnetosheath and magnetopause boundary layer on re-entry to the magnetosphere. The traversals on this occasion were caused by the arrival of an intense CME at the Earth, which produced a large compression of the magnetopause and high magnetic activity. The magnetopause traversals represent an unprecedented data set, allowing detailed analysis of the local magnetic structure (gradients) and dynamics of the magnetopause boundary. By performing minimum variance analysis (MVA) on the magnetic field data from all four spacecraft, we demonstrate that the magnetopause was planar on the scale of the spacecraft separation scales and that the transverse scale size of the magnetopause boundary layer was $1000-1100 \mathrm{~km}$. We also show that the motion of the boundary (defined by the magnetic shear at the current layer), is changing over the sequence of spacecraft crossings so that acceleration of the magnetopause can be very high in this region of the magnetosphere. Indeed, the magnetopause speed reaches the order of $300 \mathrm{~km} / \mathrm{s}$ in response to the arrival of the interplanetary shock. Using MVA coordinates, we have identified a number of magnetospheric and magnetosheath FTE signatures, which are sampled simultaneously by all spacecraft at different distances from and on either side of the magnetopause. The signatures show a variation of scale with distance from the boundary.
\end{abstract}

Key words. Magnetospheric physics (magnetopause, cusp and boundary layers) Space plasma physics (discontinuities;

Correspondence to: M. Dunlop (m.dunlop@ic.ac.uk) magnetic reconnection)

\section{Introduction}

The Cluster mission is currently providing the first coordinated data set of multi-point measurements in the near Earth environment. The four Cluster spacecraft were launched in pairs on two Soyuz rockets on the 16 July and the $9 \mathrm{Au}-$ gust and were all successfully placed into their polar orbits. The mission is designed to operate with an eccentric (4 $\left.R_{E} \times 19.6 R_{E}\right)$ inertial orbit, at $90^{\circ}$ inclination, and with a line of apsides chosen to optimise coverage of the Earth's dayside, northern cusp. Throughout a year, the orbit therefore covers the dayside magnetosphere and solar wind, although, typically only high latitude crossings of the dayside magnetopause occur, and the night-side magnetosphere and magnetotail, crossing the plasma. The individual spacecraft orbits have slightly modified elements but with identical orbital periods, so that they fly in an evolving configuration which repeats every orbit (apart from minor perturbations). Initial manoeuvres established the spacecraft configuration to correspond to that of the dayside science phase. This forms a regular tetrahedron at the exterior cusp location near noon local time, with a spacecraft separation scale size of $\sim 600 \mathrm{~km}$. Because the apogee orientation lies along noon and midnight local time in March and September, respectively, it has a tilted aspect with respect to GSE (or GSM) coordinates.

Full science coverage of the dayside magnetosphere and solar wind began on 1 February 2001. Prior to this time, all spacecraft and payload had undergone a comprehensive set of commissioning procedures and had also taken limited science data during this period. Following initial checkout procedures, all magnetometers comprising the FGM exper- 
iment have been working nominally, and have been extensively tested throughout the spacecraft commissioning period. (see Balogh et. al., this issue, for a description of the operational aspects of the FGM investigation and (Balogh et al., 1993; Balogh et al., 1997) for technical descriptions of the instruments). All FGM instruments have taken data throughout commissioning, which began with apogee of the Cluster orbits lying in the Earth's magnetotail, at midnight local time. Subsequently, inertial precession moved apogee across the magnetotail to the dusk-side flank of the magnetosphere, with the spacecraft crossing through the northern and southern magnetospheric lobes. The spacecraft first exited the magnetosphere on 9 November 2000, during a period of unusually high solar wind activity and during November and early December, the spacecraft skimmed the night-side magnetopause and magnetosheath, near apogee. During December and January, the orbit moved into the dayside magnetosphere, through the dusk flank, and provided a large number of high latitude crossings and extensive magnetosheath coverage.

We focus here on observations taken by the four spacecraft near the magnetopause and concentrate on a series of crossings which occurred before and after an intense compression of the magnetosphere, associated with a large change in solar wind dynamic pressure. The magnetopause crossings and near magnetosheath observations are discussed here in terms of recognisable features, previously reported in the literature. We first describe the whole day of observations, in terms of the location on the magnetopause, the local magnetosheath and solar wind conditions, for context. We then describe each main magnetopause encounter in turn to demonstrate the changing magnetopause character and the spacecraft location relative to the boundary structure on both sides of the magnetopause.

There have been few detailed studies of the flank region of the magnetopause because of the, often complicated, form of the boundary and the variety of magnetosheath properties. Previous studies have tended to concentrate on the subsolar region (e.g. Berchem and Russell, 1982; Russell, 1995; Paschmann et al., 1985, 1986; Phan and Paschmann, 1995), and surveys of magnetopause crossings have tended to omit the flank region (Paschmann, 1986; Phan et al., 1994). Although a number of studies have been reported using several spacecraft which happened to be favourably located at large (a few $R_{E}$ ) distances in the past, multipoint observations of the magnetopause layer and related phenomena on the mesoscale have primarily arisen from dual spacecraft, ISEE1 and 2 (Russell and Elphic, 1978) and AMPTE IRM and UKS (Bryant et al., 1985) measurements (e.g. Elphic, et al., 1988, 1990, 1995). Both these missions, however, were equatorial and sampled the LLBL (Low Latitude Boundary Layer) on the dayside. For the work discussed here, the unusual nature of the external solar wind conditions and the enhanced measurement capability provided by the four point Cluster data, makes it an important event to study.

\section{Overview and data}

The first encounters of the Cluster spacecraft with the magnetopause and traversal into the magnetosheath, on 9 and 10 November, followed the arrival of an intense CME at the Earth. This event resulted from the onset of a solar storm two days earlier the details of which are reported elsewhere (Boralv et al., 2001). Although the spacecraft were already in the magnetosheath during the end of 9 November (while at apogee), the sequence of magnetopause encounters occurred on 10 November, as the spacecraft re-entered the magnetosphere on the southern leg of the orbit. We note that on this day a number of other instruments, including the plasma ion instrument, were not taking data. FGM data from all four Cluster spacecraft are shown in Fig. 1. The data are shown as GSE Cartesian components together with the field magnitude, with all spacecraft traces superimposed to highlight any differences in the measured field. In all time series plots, the colour scheme used (here and in the subsequent plots) is: spacecraft 1 (black), 2 (red), 3 (green) and 4 (magenta). It should be noted that a number of commissioning tests were being carried out on spacecraft 1 (black trace) at times during this day, producing a number of gaps in the science data. Most of the key magnetopause crossings, however, are covered by continuous data. During normal operation, the instruments are commanded to provide primary sensor data at $22.4 \mathrm{~Hz}$ in the spacecraft normal mode and $67 \mathrm{~Hz}$ in the spacecraft burst mode. These data are filtered and re-sampled on board from an internal digital sampling rate of $202 \mathrm{~Hz}$. The magnetometers were operating in normal mode during this day. For illustration here, we have used $1 \mathrm{~s}$ averages of the data, but, where appropriate, the boundary analysis has been performed using full resolution data.

Before discussing the nature of the magnetic field measurements in Fig. 1, it is useful to note the spacecraft location, as shown in Fig. 2. Figure 2a shows a projection of the inbound leg of the orbit into the $X, Y_{\mathrm{GSM}}$ plane, for spacecraft 1, and Fig. 2b shows the corresponding plot for $Y$, $Z_{\mathrm{GSM}}$. The time interval for the whole orbit leg corresponds to the whole of the day. Each hour is marked by filled circles at intervals along the orbit. Also shown on the orbit are the spacecraft configurations at intervals, projected into the GSM plane. Spacecraft 1 is shown attached to the orbit and the other spacecraft are shown with their corresponding colours in their relative positions at the same time. The size of the spacecraft separation vectors have been scaled up by a factor of 20 (so that a length on the plot of about $2 R_{E}$ is equivalent to an actual separation of $\sim 600 \mathrm{~km})$. During the time period of the observations (00:00-09:00 UT), spacecraft 2 and 3 lie almost parallel to the plane of the figure while spacecraft 1 is separated by about $150 \mathrm{~km}$ northward and spacecraft 4 , about $650 \mathrm{~km}$ southward.

In Fig. 1, all spacecraft are therefore located initially near apogee, on the southern leg of the orbit. They lie just south of the ecliptic plane $\left(X, Y_{\mathrm{GSE}}\right)$, at about 19:00 LT (local time), and lie in the magnetosheath from the start of the day. Even at apogee, for this orientation of the orbit (see Fig. 2), the 

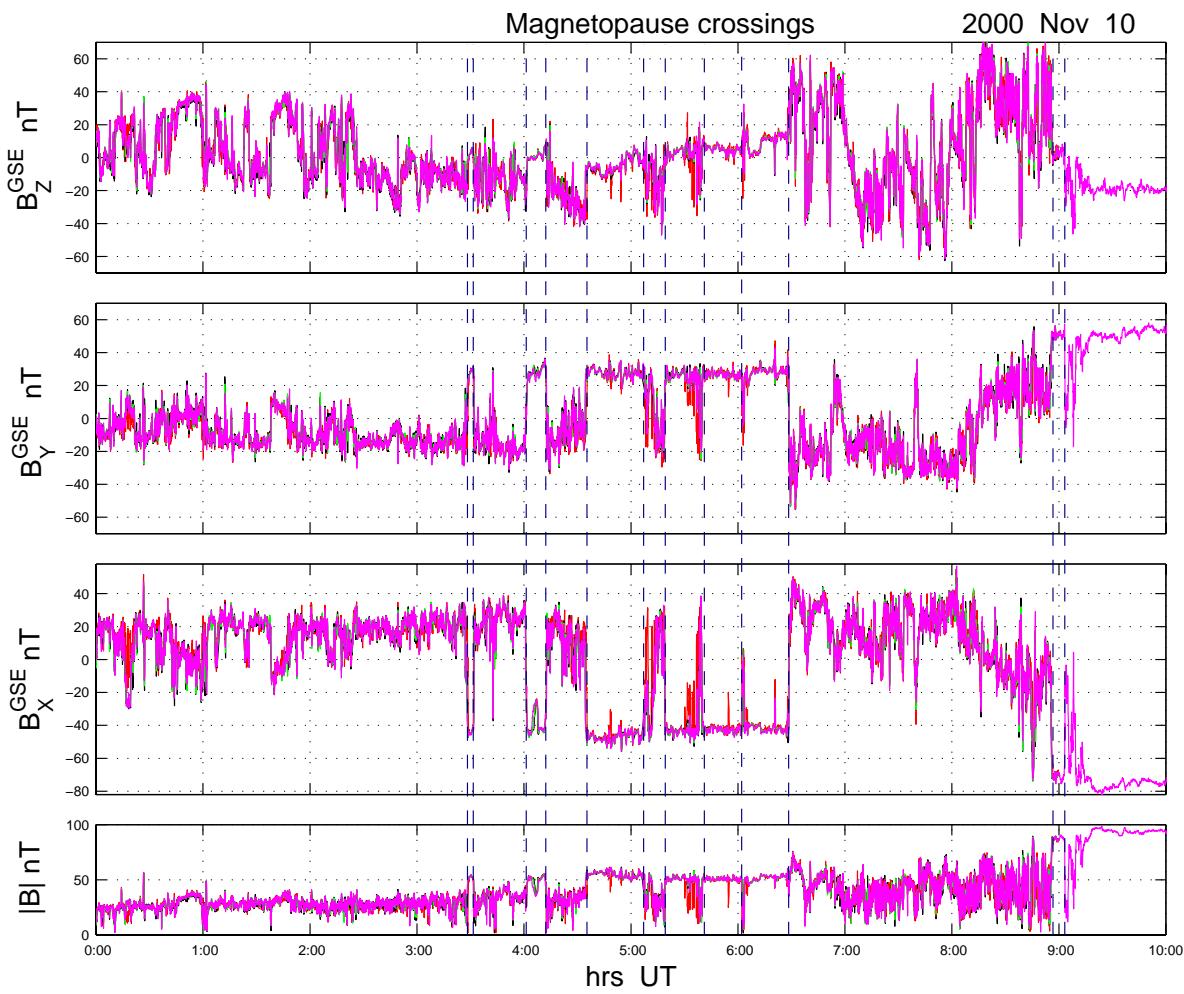

Fig. 1. Four-spacecraft, calibrated FGM data taken on 10 November 2000. The data are expressed in GSE coordinates and the field magnitude is given in the bottom panel. The spacecraft traces are represented as individual colours (s/c 1-black, s/c 2-red, s/c 3-green, s/c 4-magenta), but normally these are hidden when the spacecraft see the same field. Inter-spacecraft comparisons show that the calibrated data agree in absolute terms to within $\pm 0.2 \mathrm{nT}$. Small scale structure, or temporal changes, between the spacecraft appear as deviations between the traces. The spacecraft are in the magnetosheath at the start of the day. An extended encounter with the magnetopause, resulting in a series of crossings (indicated by dashed vertical lines), occurs between 03:30-06:30 UT. Following this, a large compression of the magnetosphere results in an extended period of magnetosheath, followed by a final re-entry into the magnetosphere. The key magnetopause crossings are indicated.

spacecraft would be expected to remain within the magnetopause for normal solar wind conditions $\left(P_{\text {ram }}<3 \mathrm{nPa}\right)$. Thus, the magnetosphere is already somewhat compressed at this time and preliminary solar wind parameters appear to be consistent with this. As the spacecraft traverse the southern leg of the orbit, inbound, they move rapidly south (with respect to GSM coordinates) to about $-10 R_{E}$ at the time of the final re-entry into the magnetosphere at $\sim$ 09:00 UT. The spacecraft would therefore be expected to skirt the form of a stationary magnetopause lying just inside the Cluster orbit. At this local time the magnetospheric field would be expected to be draped substantially tailwards with only a small $B_{Z}$ component (see discussion below on Fig. 3). At about 03:30 UT, however, the spacecraft re-encounter the magnetopause, which appears to have moved outwards, and begin a sequence of shallow crossings of the boundary layer until 06:30 UT. After 06:30 UT the spacecraft appear to move into deep magnetosheath conditions (see also a companion paper, Lucek et al., this issue).

Preliminary solar wind data (WIND, Acuña, et al., 1995) show an extremely large increase in solar wind dynamic pressure, $\left(P_{\text {ram }}>20 \mathrm{nPa}\right)$ at $\sim 06: 30 \mathrm{UT}$ (allowing for convection in the solar wind), behind a strong interplanetary shock.
Although WIND was situated on the dawn side of the SunEarth line, this solar wind signature is also seen at Geotail at about 06:27 UT, located just upstream $\left(\sim 18 R_{E}\right)$ of the magnetosphere near the sub-solar point. Most ground based instruments see signatures associated with the magnetospheric compression and subsequent relaxation at $\sim$ 09:00 UT (Boralv, et al., 2001). We therefore associate the exit of the spacecraft into the magnetosheath at $\sim 06: 30$ UT with an intense compression of the magnetosphere following arrival of the pressure pulse behind the interplanetary shock. Both the preliminary WIND and Geotail data also indicate that the IMF lies predominantly in the ecliptic, along the Parker spiral direction, or has an unstable $B_{Z}(\mathrm{GSE})$ signature (not exceeding $\sim 5 \mathrm{nT}$ before the interplanetary shock). The IMF is southward for only brief intervals. In order to obtain an approximate, expected, magnetopause location we only use therefore pressure scaling to fit the magnetopause location (see Dunlop et al., 1999). For simplicity here, we compare magnetopause location to the geometric model of Sibeck et al. (1991). The dashed line shown in Fig. 2a represents a cut of the model magnetopause through the intersection with the orbit (at $\sim 08: 55 \mathrm{UT}$ ), and corresponds to $\sim 6 \mathrm{nPa}$, for a cut at $-10 R_{E}$. In fact, the Geotail data indicate that the dynamic 

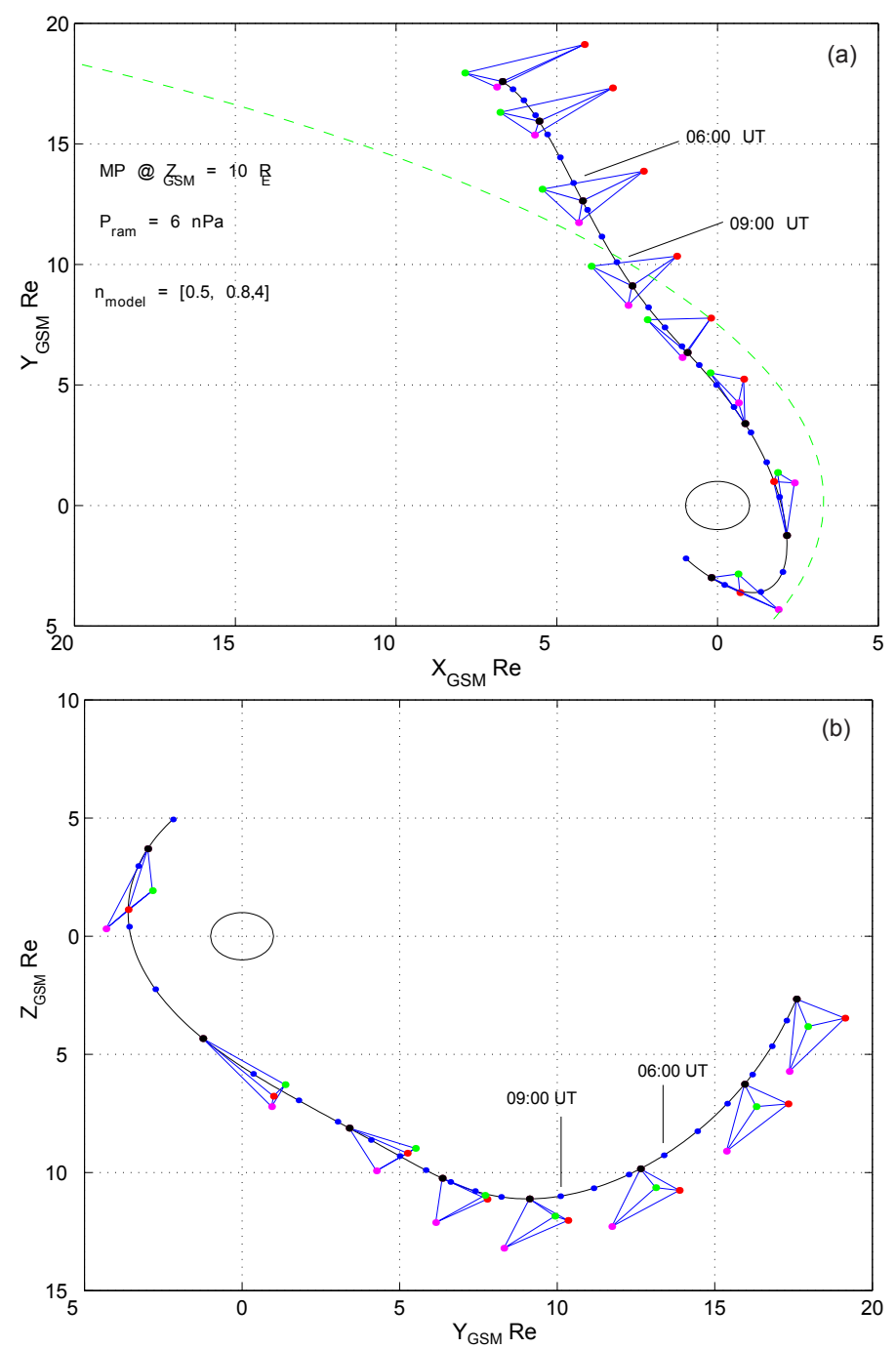

Fig. 2. (a) Projected plot of the Cluster orbit (spacecraft 1) into $X, Y_{\mathrm{GSM}}$ coordinates and model magnetopause location, fitted to the crossing near 09:00 UT. The configuration of the spacecraft is shown at intervals along the orbit, with the separations scaled by a factor of 20. As can be seen by Fig. 2b, spacecraft 2 and 3 lie almost parallel to the $X, Y$ plane, with spacecraft 1 above and spacecraft 4 below. The magnetopause is shown as a cut through the intersection with the orbit. The model normal and the ram pressure used is also shown (b) As for Fig. 2a, but in the $Y, Z_{\mathrm{GSM}}$ plane.

pressure at the sub-solar point remains somewhat higher than this (in excess of $15 \mathrm{nPa}$ ) just after 09:00 UT, suggesting that not all the solar wind pressure is transmitted to these high, southern latitudes. The figure also indicates the model normal at the crossing position.

Locating the magnetopause just outside of (or at) the spacecraft positions between 03:30-06:30 UT corresponds to a solar wind dynamic pressure of $4-5 \mathrm{nPa}$, which is close to that observed at the sub-solar point by Geotail prior to the arrival of the pressure pulse at the interplanetary shock. The effect of the IMF- $B_{Z}$ on the shape of the magnetopause at this local time and latitude of Cluster is not well known, since most data sets have used available data predominantly from low-latitude spacecraft. Studies of the shape and location of the magnetopause boundary, including the effect of IMF- $B_{Z}$, have been reviewed by Fairfield (1995) and Shue et al. (2000). Since we use the model boundary for guidance only, a detailed consideration of this effect is not critical to the discussion here. The effect of a 5 nT IMF- $B_{Z}$ on the flaring of the magnetopause on the flanks could be of order $1 R_{E}$ (Shue et al., 1998) and this is included in the pressure uncertainty quoted above $(\sim 1 \mathrm{nPa})$. After the arrival of the interplanetary shock, the IMF- $B_{Z}$ fluctuations increase slightly but predominantly remaining within $\pm 10 \mathrm{nT}$, as often northward as southward. The effect of magnetopause shape on the model normal is much smaller than the deviations in actual normals quoted below.

Figure 3 shows the time period containing both the main boundary layer encounter and the final entry into the magnetosphere, in polar GSE coordinates, for the data from spacecraft 4. The T89 magnetospheric field model (Tsyganenko, 1989), corresponding to the high activity conditions observed 

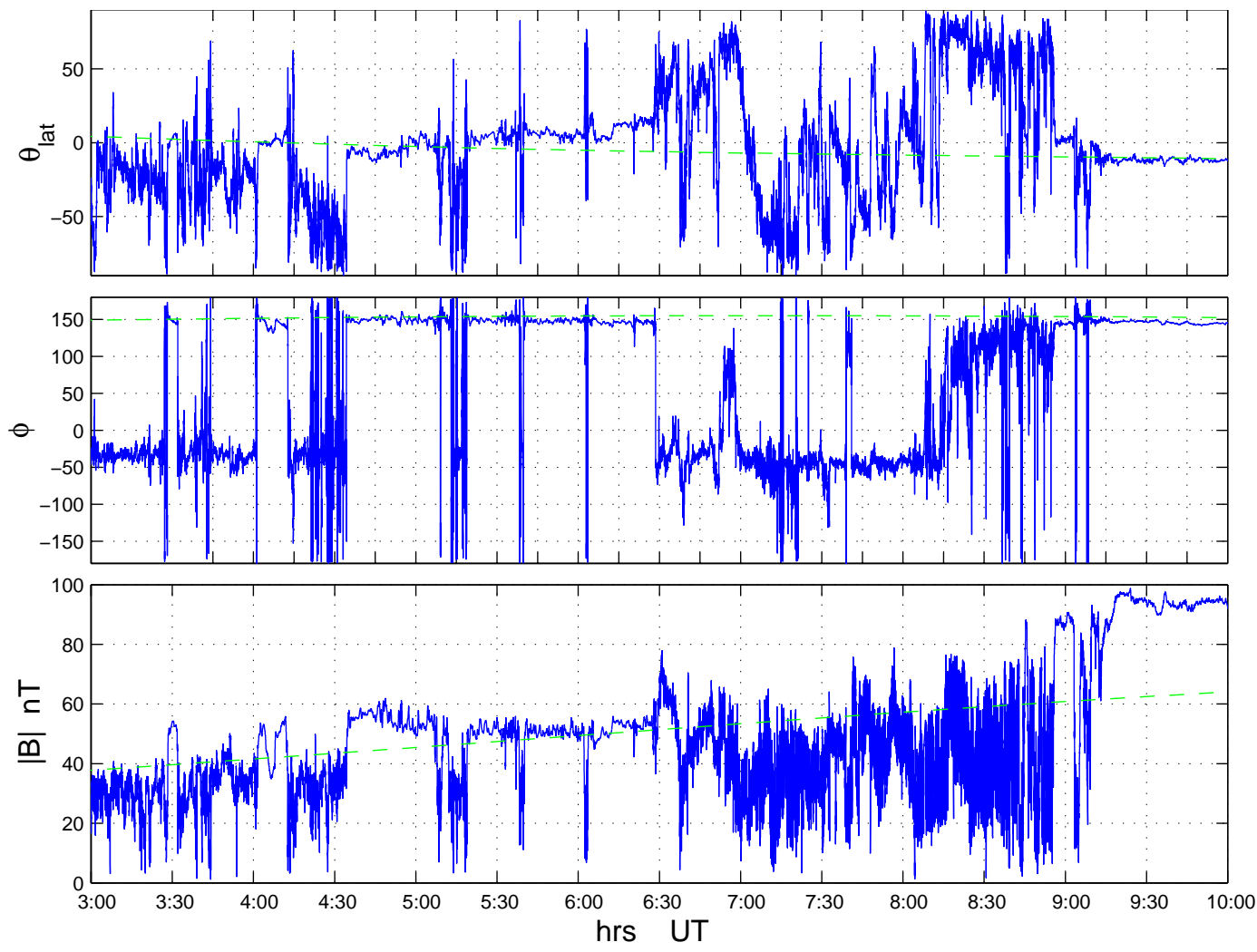

Fig. 3. Plot of the magnetic field measured on spacecraft 4 in GSE polar coordinates. The model field, computed using the Tsganenko-89 model for an extreme activity level $\left(K_{P}=6\right)$, is shown as dashed lines. The measured field shows high compression after the 09:00 UT crossing. The orientation of the model field fits the data very well, except just before the 06:30 UT crossing.

during this event has been added for comparison. The high $K_{P}=6$ value corresponds to the best fit to the field orientation, defined by the polar angles, during this data interval. Clearly, the high magnetic field magnitude after 09:00 UT, in comparison to the model field, represents a highly compressed magnetosphere and is consistent with there having been a sustained, large increase in solar wind dynamic pressure after 06:30 UT. When the spacecraft are measuring magnetospheric field (for the intervals we identify), the field lies close to themodel field (dashed line) throughout each interval. This direction corresponds to a twisted field configuration at the southern, night-side location, where the field direction points predominantly tailward in a draped orientation. Although the magnetospheric field directions near the magnetopause follow the model closely, high values of magnetic field intensity are not modelled closely by the T89 model in the flank regions. By contrast, the magnetosheath field shows a variation broadly in line with preliminary IMF behaviour at the WIND spacecraft. After about 02:30 UT it shows a variable north/south orientation, predominantly close to the Parker spiral (with $B_{X}$ positive) and this results in high magnetic shear across the magnetopause for the crossings between 03:30-06:30 UT. After about 08:00 UT the magnetosheath field turns predominantly northward, corresponding to the IMF, and away from the Parker spiral direction just before the last crossing. This change in orientation significantly reduces the local shear across the magnetopause for the last encounter (at 08:55 UT).

\section{Magnetopause encounters}

In order to investigate the character of the magnetopause and its dynamics in response to the changing conditions, we select four encounters with the magnetopause. The results of MVA (minimum variance analysis, (Sonnerup and Cahill, 1967)) of each crossing are shown in Table 1. The table shows the date and key time of each crossing, the orbital position of spacecraft 1 , the mean boundary normals for each crossing and the mean velocity of the boundary along the normal. The column headed 'MP' labels each encounter $1-4$, numbered backwards in time. In the Table, we have shown only the average over the individual spacecraft normals and the average of the magnetopause velocity between each spacecraft pair. The velocity is calculated from the individual crossing times at each spacecraft and the projected separation vector for each pair of spacecraft (see Bauer et al., 2000, for an application of inter-spacecraft timing methods using AMPTE IRM/UKS data). We comment on the differences between each spacecraft for the individual crossings below. 
Table 1. Boundary analysis results

\begin{tabular}{|c|c|c|c|c|c|c|c|c|c|c|c|c|}
\hline Date & UT & MP & LT & $\operatorname{rr}(\mathrm{Re})$ & $\mathrm{x}$ & $\mathrm{y}$ & $\mathrm{z}$ & & $\mathrm{n}_{a v}$ & & $\lambda_{2} / \lambda_{3}$ & $\mathrm{v}_{n}$ \\
\hline 10 Nov. 2000 & 08:55:30 & 1 & $18: 51$ & 15.27 & -3.17 & 13.92 & -5.41 & 0.56 & 0.77 & -0.29 & 15.00 & 34.8 \\
\hline 10 Nov. 2000 & $06: 28: 31$ & 2 & 19:01 & 16.65 & -4.28 & 15.52 & -4.23 & 0.60 & 0.74 & -0.30 & 12.00 & -124.9 \\
\hline \multirow{2}{*}{10 Nov. 2000} & 06:02:00 & 3 -in & $19: 03$ & 16.91 & -4.52 & 15.78 & -4.05 & 0.51 & 0.85 & -0.15 & 3.00 & -17.3 \\
\hline & 06:03:40 & 3-out & $19: 03$ & $16: 91$ & -4.52 & 15.78 & -4.05 & 0.51 & 0.85 & -0.15 & 3.00 & 19.9 \\
\hline 10 Nov. 2000 & 05:40:00 & 4 & $19: 06$ & 17.21 & -4.80 & 16.11 & -3.73 & 0.14 & 0.56 & -0.82 & 5.00 & 44.3 \\
\hline
\end{tabular}

Although there are small changes in magnetopause orientation between encounters, the normals are broadly consistent (they agree to within a few percent in direction) and, except for the encounter at 05:25-05:40 UT, are in line with the model magnetopause at each location. Except for the fast crossing at $\sim 06: 30 \mathrm{UT}$ (see below), the normals are all stable (to choice of analysis interval), typically with high eigenvalue ratios $(>5)$ between intermediate and minimum eigenvalues. Statistical errors (as defined in Sonnerup and Scheible, 1998) are small. The evolution of the boundary orientation follows the slow change in spacecraft location and the individual boundary normals at each spacecraft show that the local boundary shape has a high degree of planarity. This fact makes the interpretation of each crossing sequence relatively easy. In the majority of cases, repeated encounters with the magnetopause boundary show nested sequences of crossing times at each spacecraft; strong support for the interpretation that the crossings are caused by in/out motion of the boundary, rather than convected structure moving passed the Cluster configuration. Such nested timing is maintained for all crossings throughout the day, although the actual order of the spacecraft encounters changes when the boundary orientation changes.

The magnetosheath field remains closely aligned to the magnetopause plane, except for some notable intervals discussed below. This suggests that, at least for the interval 03:30-06:30 UT, the Cluster configuration remains close to the magnetopause and is consistent with small, but changing, motion of the magnetopause during this period. The computed velocities confirm this, noting that we use the convention that outward motion of the magnetopause, along the (outward) normal to the boundary, is positive. Crossing 1 occurs after the magnetopause has stabilised and is slowly reexpanding, since it shows a clear outward motion at all the spacecraft. The corresponding normal directions at crossing 1 are closely aligned to the model normal. The encounter follows the large magnetospheric compression, which occurred immediately after crossing 2, where subsequently, the spacecraft moved substantially south with respect to the GSM equator and followed the magnetopause inwards until 09:00 UT. Crossing 2 shows a high inward velocity, consistent with the onset of a large compression of the magnetopause. Crossing 3 is chosen because it shows a rapid but simple reversal in boundary velocity which corresponds to a similar low speed for both the inwards and outwards motion of the boundary, consistent with a small amplitude depres- sion of the magnetopause. Crossing 4 is also consistent with outward motion.

Corresponding to the table, each crossing is discussed below in more detail, in reverse time order.

\subsection{The crossings between 08:30 and 09:00 UT}

Figure 4a shows the interval around the final re-entry of the Cluster spacecraft into the magnetosphere for all four spacecraft. In fact, as shown by Fig. 1, there are more brief crossings back into the magnetosheath after 09:00 UT, after which the spacecraft remain in the magnetosphere. Only data from spacecraft 4 , however, were recovered for these later times. The data are expressed in MVA coordinates, with ' $N$ ' along the minimum variance direction, ' $M$ ' along the intermediate variance direction and ' $L$ ' along the maximum variance direction. The coordinates have been determined for each spacecraft separately by performing MVA across the magnetopause. The boundary normals (minimum variance direction) at each spacecraft are, however, closely aligned (within $1 \%$ ) and are very stable with a high separation of the minimum and intermediate eigenvalues. The profiles of each spacecraft show a high degree of agreement, but also show evidence of small-scale structure. The gross (low frequency) field configuration around the magnetopause, and the implied planar geometry of the magnetopause, are therefore maintained on the scale of the spacecraft separations (which range from $\sim 400$ to $\sim 1000 \mathrm{~km}$ ).

The $B_{N}$ component shows fluctuations about zero, suggesting that the magnetic alignment is tangential across the boundary and that the magnetosheath field remains closely aligned to the magnetopause. This is confirmed by the boundary analysis. Figure $4 \mathrm{~b}$, for instance, shows a scatter plot of the GSE angles of the magnetic field for an interval around the magnetopause. The data are from spacecraft 4 and the MVA normal is quoted on the plot. Two curves are drawn through the points (see Dunlop and Woodward, 1998 and Dunlop et al., 1999 for a description). The green curve represents a fitted planar field geometry (where the magnetic field vector lies in different directions but on a plane) to the points and the red curve the equivalent plane defined by the MVA normal. The two curves agree to within the statistical error and, together with $\theta_{b n}$ (the out of plane angle and lower panel in Fig. 4b), confirm tangential field alignment across the magnetopause. As indicated in Fig. 4a, there is a brief, earlier encounter with the magnetopause, between 08:44:30- 

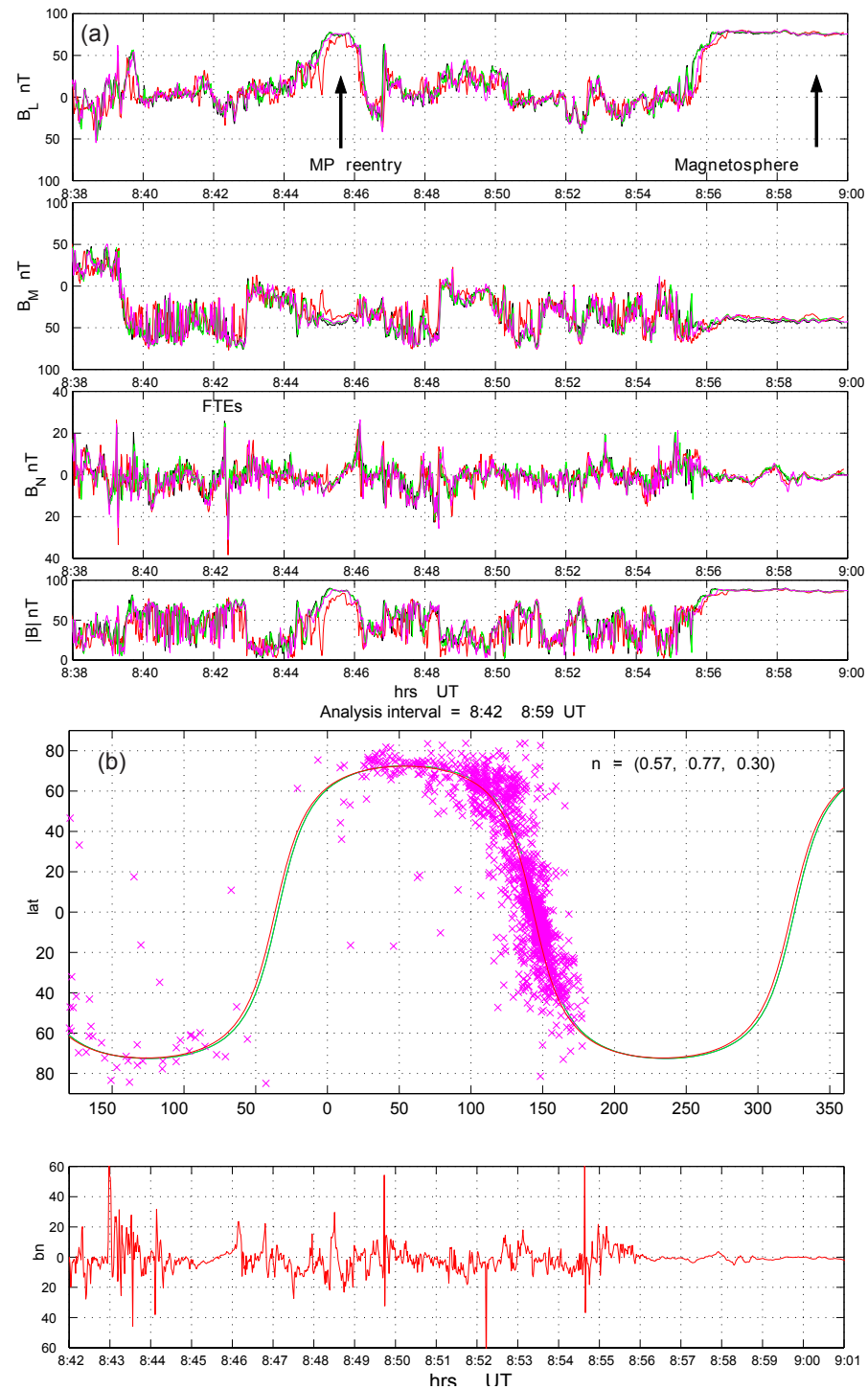

Fig. 4. (a) Interval of data in minimum variance coordinates around crossing 1, as in Table 1. MVA is performed for each spacecraft separately in order to express the data in LMN coordinates. The interval is chosen to show both the brief re-entry into the magnetosphere and two clear FTE signatures in the magnetosheath. The analysis is performed at high resolution $(22.4 \mathrm{~Hz})$. (b) Result of the boundary analysis, as described in the text, where the interval plotted refers to the scatter plot. The MVA was performed across the MP at 08:56 UT.

08:46:30 UT, suggesting that the spacecraft remain adjacent to the magnetopause, but in the magnetosheath, around this time. The traces show a larger time delay at 08:44:30 UT than at 08:46:30 UT. Since the normal direction does not change for the inbound or outbound crossing, the nested signature of the re-entry (most clear for the red trace), therefore, indicates a slower entry into the magnetospheric boundary, than the exit. Note that throughout the plot, the black and green traces remain close together, reflecting the fact that these two spacecraft (1 and 3) remain closely parallel to the magnetopause plane. There are a number of short turnings in the $B_{N}$ component throughout the period shown but two strong, bipolar signatures with FTE-like signatures (Russell and Elphic, 1979) are notable at 08:39:15 and 08:42:20 UT. These signatures stand out because of the similarity at each spacecraft (which sets the overall scale size to be of order $1000 \mathrm{~km}$, or greater) and their close coincidence in time. At high resolution, the time shifts between each spacecraft trace suggest a convection speed of $700-900 \mathrm{~km} / \mathrm{s}$, parallel to the magnetopause and a corresponding scale (along the flow) over the signature of $4000-5000 \mathrm{~km}$. This is consistent with the expected magnetosheath flow for the solar wind conditions at this time. In detail, there is structure on the spacecraft separation scale, particularly within the enhancements in field strength. At high time-resolution, for the first event (at 08:39:15 UT), spacecraft 1 and 3 both show a dip in $|B|$ in the centre of the event, while spacecraft 2 and 4 do not (not clear from Fig. 4a). As referred to above, the orientation of the magnetopause is such that spacecraft 1 and 3 lie almost parallel to its plane, with spacecraft 2 and 4 lying further out 

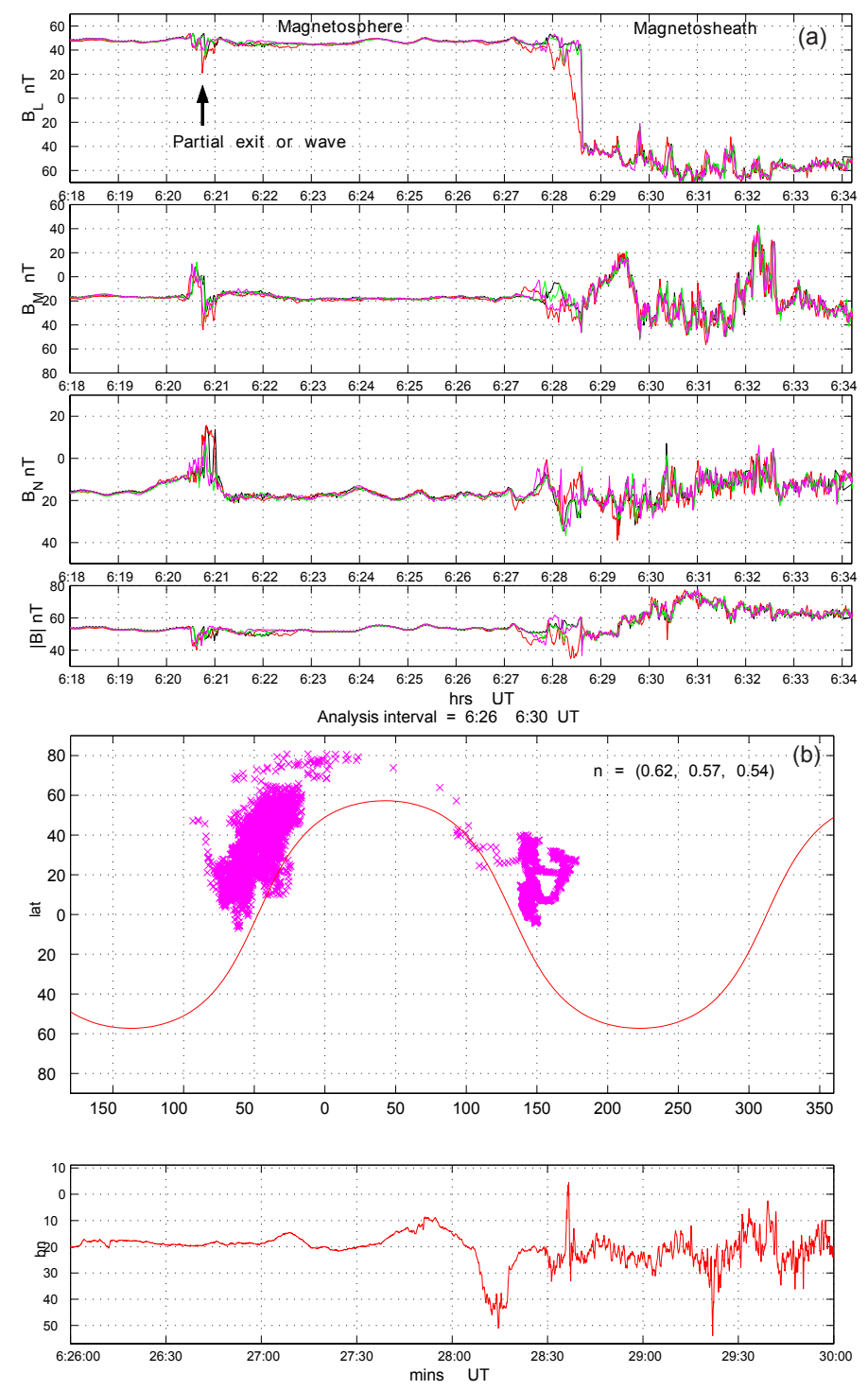

Fig. 5. (a) As for Fig. 4a, but for crossing 2. (b) As for Fig. 4b, but for crossing 2. The planar fit to the scatter plot is not shown.

(spacecraft 4 is also south of the others). For a cylindrical flux tube moving along the magnetosheath flow, therefore, spacecraft 1 and 3 would cut through the structure similarly.

At the magnetopause crossing at 08:56 UT, it is clear from the $B_{L}$ component that spacecraft 1 and 3 pass through the magnetic shear almost at the same time, followed by spacecraft 4 and then spacecraft 2 (a similar sequence occurs at the brief entry at 08:44:30 UT). The nature of this crossing at all the spacecraft is consistent with the view that the magnetosphere is undergoing expansion and recovery, following the large compression earlier. Using the independently determined normals, which demonstrate a high degree of planarity over the spacecraft tetrahedron, we can compute projected speeds along the normals for each spacecraft pair from the inter-spacecraft timing and separation vectors. We do not describe the full technique here, since it is described elsewhere in detail but it is worth noting the following points. The calculation rests on each spacecraft measuring similar substructure, which produces stationary features in the timeseries. Since, in general, significant intervals of data around the boundary will contain features representing both time dependence and spatial structure, this means that the use of correlation techniques for the timing usually fail. Moreover, constant velocity can almost never be assumed. Although the boundary here is relatively stable, we find that the velocity changes smoothly from $20 \mathrm{~km} / \mathrm{s}$ to $45 \mathrm{~km} / \mathrm{s}$ as it passes the spacecraft array. We estimate that the boundary layer extends over about $30 \mathrm{~s}$, giving a thickness of $\sim 1000 \mathrm{~km}$, corresponding to the mean speed in Table 1.

\subsection{The crossings at 06:30 UT}

Figure 5a shows an overview of the magnetopause crossing at the time of arrival of the interplanetary shock and increase in solar wind dynamic pressure in the same format as for Fig. 4a. At a convection speed of up to $900 \mathrm{~km} / \mathrm{s}$, the pressure 
increase could arrive at Cluster just before the crossing while the spacecraft were still in the magnetosphere. The main characteristic of this crossing is the speed of traversal, which results in almost simultaneous exit times at spacecraft 1, 3 and 4. There is also significant structure (on the scale of the spacecraft separation) within the magnetospheric boundary layer (06:27:50-06:28:40 UT) and the separation of the red trace for spacecraft 2 suggests rapid acceleration between this and the other spacecraft.

MVA analysis of the boundary and adjacent region gives the normals, quoted in Table 1, which are consistent with the model normals at this position and all spacecraft agree to within 5\%. However, the complexity of the magnetopause layer itself, which appears to contain an embedded FTE ( $B_{N}$ signature) or boundary wave, produces slightly different normals. The traces for $B_{N}$, in Fig. 5a, show deviations between the spacecraft, but all have a consistent component of $\sim 20 \mathrm{nT}$, indicating that the current layer has the form of a rotational discontinuity. Figure $5 \mathrm{~b}$ shows the high-resolution analysis for spacecraft 3 in the format of Fig. $4 \mathrm{~b}$. The evidence from the scatter plot suggests that the underlying magnetopause plane has a much smaller $Z_{\mathrm{GSE}}$ component than suggested by the MVA normal. Such an orientation places the magnetopause almost parallel to the spacecraft plane defined by spacecraft 1, 3 and 4 . Spacecraft 2 lies almost perpendicular to this plane at a separation of about $650 \mathrm{~km}$. Such a local orientation of the magnetopause would be consistent with a sudden large compression, driving the magnetopause inwards.

Although this alignment of the spacecraft and magnetopause planes means that the computed speed of the boundary is sensitive to the normal direction, the mean velocity from the spacecraft 2 timing can be accurately found and is $45 \mathrm{~km} / \mathrm{s}$. Moreover, the only consistent analysis of the motion suggests that the boundary speed increases to over $300 \mathrm{~km} / \mathrm{s}$ at spacecraft 1 so that there is a rapid acceleration of the magnetopause inwards. It is again true that spacecraft 1 and 3 sample very similar fields, while spacecraft 2 shows distinct structure. Spacecraft 4 shows almost the same profile as that for 1 and 3 after 08:28:35 UT, corresponding to the outer current layer and in line with the boundary orientation implied above. Spacecraft 2 sees different structure through the boundary and transition layers, but all spacecraft show signatures that are consistent with a high shear boundary (Paschmann et al., 1986; Phan et al., 1994). The transition layer again extends over $30 \mathrm{~s}$ and the perpendicular scale of the boundary layer at the velocity determined from spacecraft 2 is, therefore, again just larger than $\sim 1000 \mathrm{~km}$.

\subsection{The crossings at 06:05-06:10 UT}

Figure 6 shows a pair of magnetopause crossings corresponding to a simple reversal in magnetopause motion (as indicated in Table 1). The magnetopause structure is almost the same for both the inward and outward crossing, so that the field profiles are reversed around 06:03 UT. The MVA normals are not so well determined for this interval, but show a slight rotation into $Y_{\mathrm{GSE}}$. Small differences between the spacecraft are within statistical error. The twin bipolar signature on the magnetosheath side of the boundary is similar to that seen in the magnetospheric layer and appears to be a standing structure with little or no time shift between the signatures. Again spacecraft 1 and 3 show similar profiles which would align the structure to the magnetosheath field (primarily along the Parker spiral). There are a number of magnetospheric FTE signatures after 06:04 UT (see below).

\subsection{The crossings at 05:40 UT}

Figure 7a shows a magnetopause encounter for which the spacecraft remain in or very near the boundary for more than ten minutes. All spacecraft exit briefly into the magnetosheath between 05:38 and 05:40 UT; and then all return into the magnetosphere as the magnetopause moves outwards for a sustained period. The MVA analysis was performed on the final set of crossings and produced stable normals to a tangential boundary (at least on the magnetospheric side), as can be seen in Fig. 7b for spacecraft 3. All normals for each spacecraft were again closely aligned $(<1 \%)$. It is apparent that the magnetopause orientation differs from the model normal and, from the later encounters, tilting predominantly into $Y_{\mathrm{GSE}}$ and $Z_{\mathrm{GSE}}$. This affects the crossing order for the spacecraft so that spacecraft 2 is no longer perpendicular to the boundary. The magnetospheric and magnetosheath fields have the same orientation and still see very similar field profiles. Spacecraft 4 , however, now has a larger separation along the normal.

On exit, however, at 05:38:15 UT, the magnetopause orientation is consistent with the model normals, so that spacecraft 2 is still the outer most spacecraft relative to the boundary orientation, showing multiple crossings of the magnetopause throughout the earlier period while the other spacecraft remain predominantly on the magnetospheric side of the boundary. During this interval (05:30-05:38 UT), therefore, the magnetopause boundary layer lies sometimes between spacecraft 2 and the other spacecraft and, on at least one occasion spacecraft 4 makes a partial exit into the magnetosheath. Spacecraft 1 and 3 always lie deeper on the magnetospheric side of the boundary. The $B_{N}$ trace shows close alignment to the magnetopause orientation and contains a number of (magnetospheric) FTE signatures. We have indicated four by dashed vertical lines. In some cases, notably the signatures at 05:35:00 and 05:35:25 UT, the same coherent signature is observed even when spacecraft 2 is in the magnetosheath. Thus, the same reconnected flux is seen on either side of the current layer. In the second case, for example, spacecraft 4 passes through the centre of the flux tube while the others lie either side of the boundary.

Spacecraft 2 is only $400 \mathrm{~km}$ from spacecraft 4 , along the normal, and the other spacecraft are less than $200 \mathrm{~km}$ separated so the FTE signature represents a structure of order or less than the scale of the overall boundary layer. This is consistent with previous studies of ISEE 1 and 2. Moreover, these magnetospheric signatures are of short duration, imply- 

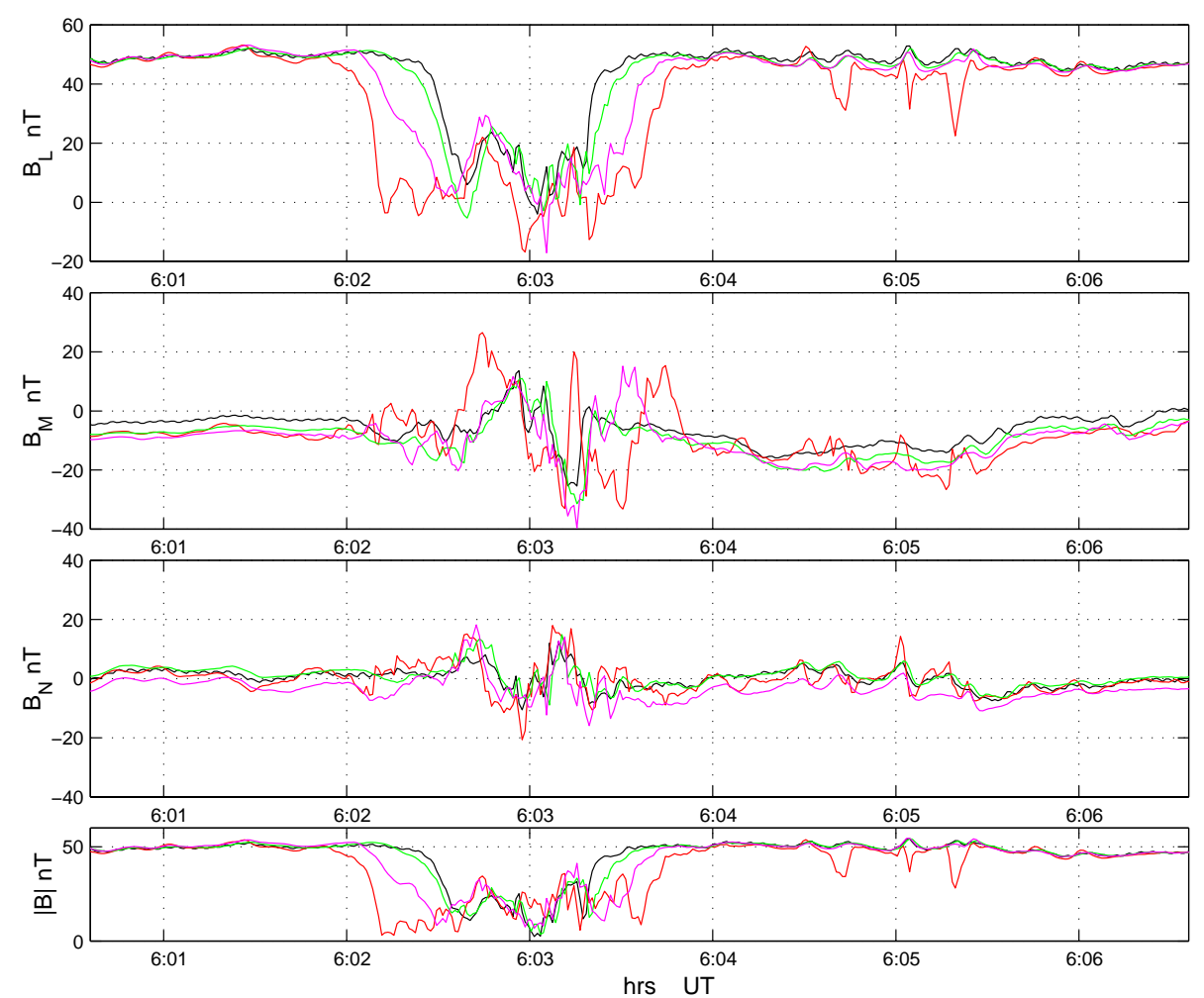

Fig. 6. As for Fig. 4a, but for crossing 3.

ing a speed along the magnetopause of $\sim 20-80 \mathrm{~km} / \mathrm{s}$, from previous estimates of their spatial scale. There is also an implication for the size of the $B_{N}$ perturbation, which appears to be always larger on the magnetosheath side of the boundary. This is true for the FTE's seen at 06:05 UT also (see Fig. 6). It is expected that the size of the signature should grow as the spacecraft cut closer to the current layer and that deeper into the magnetosphere the signature weakens.

\section{Conclusions}

We have discussed the four spacecraft magnetic field data from the Cluster mission for the first day of crossings of the magnetopause by spacecraft . We have focussed on four distinct encounters, each with particular characteristics, but in the context of the magnetospheric conditions applying to the whole day. The two main intervals of magnetospheric field, before and after the arrival of the interplanetary shock, also have associated ground signatures which, are discussed elsewhere (this issue). The whole set of crossings, measured simultaneously at the four spacecraft, in general result in a sequence of crossing times which is consistent in all cases with large scale (but often small amplitude) motions of the magnetopause. The individual ordering, in which the spacecraft cross the boundary at each crossing, and indeed the timing of other comparative features in the field profiles, have to be understood in terms of the orientation of the magnetopause with respect to the spacecraft configuration. For this event, it is demonstrated, by direct application of MVA across the magnetopause, that the geometry of the magnetopause is highly planar over the spacecraft array. The mean magnetopause orientation changes slowly over the day and is observed to change the crossing order of the spacecraft. A planar configuration makes inter-comparison of the magnetic field signatures more straightforward but also allows the motions of the magnetopause to be determined unambiguously for the first time.

We have been able to show that in nearly all cases the magnetopause motion is constantly changing and in particular varies over the sequence of spacecraft crossing times. Magnetopause speeds along the normal for the sequence of in/out crossings between 03:30-06:30 UT range from $17-44 \mathrm{~km} / \mathrm{s}$; the arrival of a pressure pulse of more than $20 \mathrm{nPa}$ produced magnetopause speeds of over $300 \mathrm{~km} / \mathrm{s}$. On the scale size of the Cluster separation distances, here ranging from 200 $650 \mathrm{~km}$ for the whole time interval, the magnetopause velocity is hardly ever constant. It is also demonstrated that the particular geometry for this day resulted in the magnetopause lying nearly planar to one face of the spacecraft tetrahedron, so that the inter-spacecraft timing is sensitive to changes in magnetopause orientation.

The scale size of the magnetopause layer was shown to be consistently (for different crossing speeds) about 1000 $1100 \mathrm{~km}$. It is important to note that, in the presence of strong sudden accelerations, an assumption of constant motion over these spatial scales (as has to be done in the case of dual spacecraft measurements) would falsely estimate the 

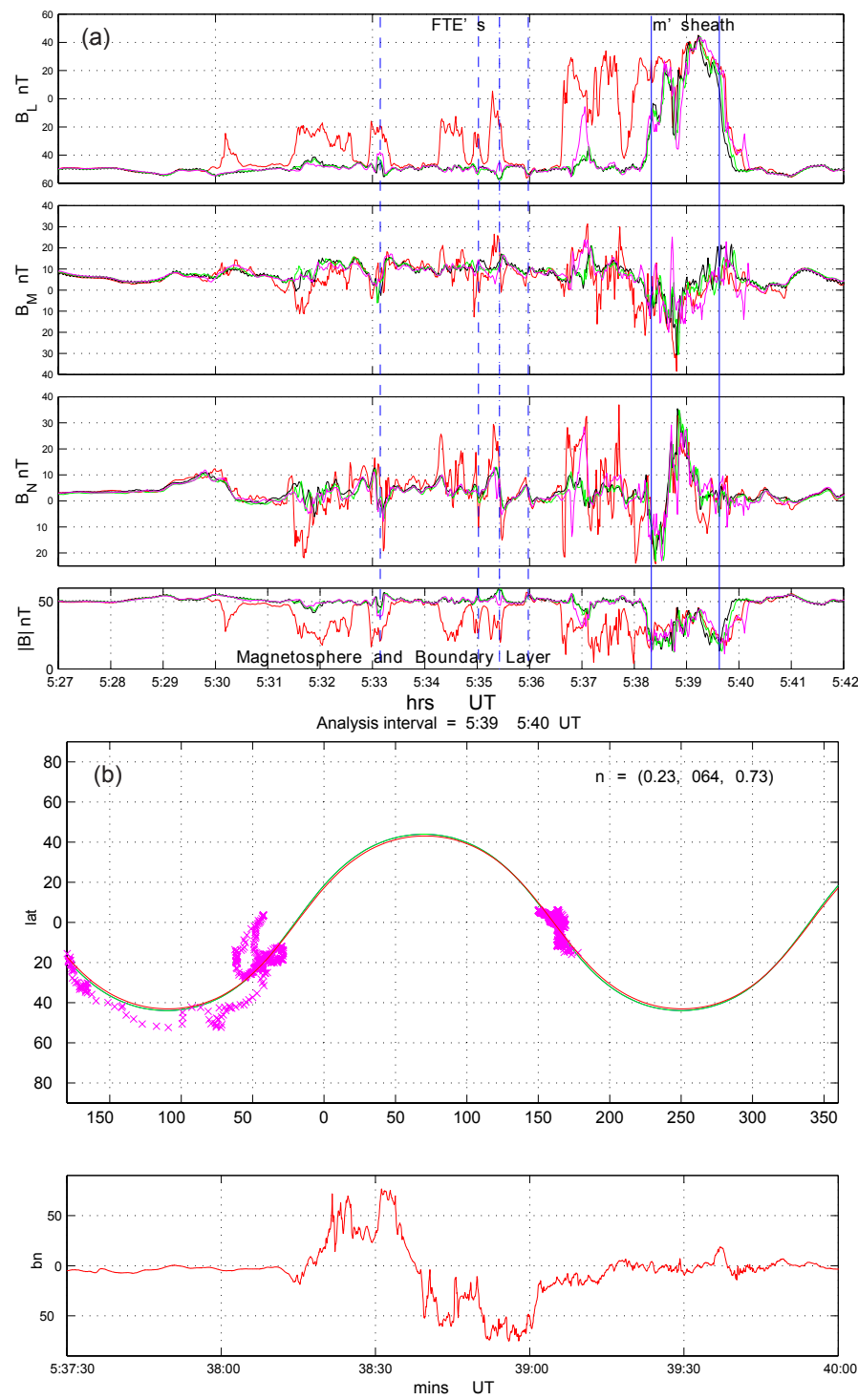

Fig. 7. (a) As for Fig. 4a but for crossing 4. The interval is chosen to correspond to the extended sequence of multiple crossings by spacecraft 2. The dashed vertical lines indicate selected FTE signatures and the short period when all spacecraft exit into the magnetosheath is shown by solid vertical lines. (b) Analysis of the boundary at 05:40 UT.

boundary thickness. Such an estimate would vary, depending on the changes in motion of the boundary. Correct use of four spacecraft information can determine, as is done here, what changes in motion occur. The perpendicular scale of embedded structures, such as FTE's, was shown to be of order or less than the magnetopause thickness. In the adjacent magnetosheath, a number of candidate FTE signatures were observed and two convected signatures, seen after the large solar wind pressure pulse, were shown to imply a magnetosheath speed of $\sim 800-1000 \mathrm{~km} / \mathrm{s}$, consistent with the solar wind speed.

For significant time intervals, the spacecraft array remained near, in, or across both the magnetosheath and magnetopause boundary layers. On a number of occasions, FTE signatures were observed by different spacecraft on either side of the magnetopause current layer. There is clear evi- dence that the scale of the signature is largest on the magnetosheath side of the boundary and has some variation with distance from the boundary. The implied flux tube motion of $\sim 100 \mathrm{~km} / \mathrm{s}$ along the magnetopause boundary suggests a parallel scale of $\sim 0.5 R_{E}$.

Acknowledgements. This work was supported at Imperial College by PPARC. Comments on the up-stream solar wind conditions were made on the basis of key parameter data held at the NASA/GSFC Space Physics Data Facility and the NSSDC, obtained via CDAweb. WIND data is made available by K. Ogilvie and R. Lepping. Geotail data is made available by L. Frank and S. Kokubun.

Topical Editor G. Chanteur thanks J. L. Burch and another referee for their help in evaluating this paper. 


\section{References}

Acuña, M., Ogilvie, K. W., Baker, D. N., Curtis, S. A., Fairfield, D. H., and Mish, W. H.: The global Geospace program and its investigations, Space Sci. Rev., 71, 5, 1995.

Balogh, A., Carr, C. M., Acuña, M. H., Dunlop, M. W., Beek, T. J., Brown, P., Fornaçon, K.-H., Georgescu, E., Glassmeier, K.-H., Harris, J., Musmann, G., Oddy, T., and Schwingenschuh, K.: The Cluster magnetic field investigation: overview of in-flight performance and initial results, Ann. Geophysicae, this issue, 2001.

Balogh, A., Cowley, S. W. H., Dunlop M. W., et al.: The Cluster magnetic field investigation: Scientific objectives and instrumentation, in Cluster: mission, payload and supporting activities, ESA SP-1159, 95, 1993.

Balogh, A., Dunlop, M. W., et al.: The Cluster magnetic field investigation, Space Science Reviews, 79, 65, 1997.

Bauer, T. M., Dunlop, M. W., Sonnerup, B. U. Ö, Sckopke, N., Fazakerley, A. N., and Khrabrov, A. V.: Duel spacecraft determinations of magnetopause motion, Geophys. Res. Lett., 27, 18351838, 2000.

Berchem, J. and Russell, C. T.: The thickness of the magnetopause current layer: ISEE 1 and 2 observations, J. Geophys. Res., 87, 2108-2114, 1982.

Borälv, E., Donovan, E., Voronkov, I., Eglitis, P., Amm, O., André, M., Balogh, A., Behlke, R., Cowley, S. W. H., and Dunlop, M., W.: The Storm of 10 November 2000, submitted to, J. Geophys. Res., 2001.

Bryant, D. A., Krimigis, S. M., and Haerendel, G.: Outline of the Active Magnetospheric Particle Tracer Explorers (AMPTE) mission, IEEE Trans. Geosci. Remote Sensing, GE-23, 177, 1985.

Dunlop, M. W., Balogh, A., Baumjohann, W., Haerendel, G., Fornaçon, K.-H., Georgescu, E., et al.: Dynamics and local boundary properties of the dawn-side magnetopause under conditions observed by equators, Ann. Geophysicae, 17, 1535-1559, 1999.

Dunlop, M. W. and Woodward, T. I.: Discontinuity analysis: orientation and motion, in: Analysis methods for multispacecraft data, ISSI Scientific Report SR-001, ESA Publications, Noordwijk, The Netherlands, p. 271, 1998.

Elphic, R. C.: Multipoint observations of the magnetopause: results from ISEE and AMPTE, Adv. Space Res., 8, 223-238, 1988.

Elphic, R. C.: Observations of flux transfer events: are FTE's flux ropes, islands, or surface waves?, in: Physics of the Magnetic Flux Ropes, (Eds) Song, Russell et al., AGU Geophysical Monograph 58, 455-471, 1990.

Elphic, R. C.: Observations of flux transfer events: a review, in: Physics of the Magnetopause, (Eds) Song, Sonnerup and Thompsen, AGU Geophysical Monograph 90, 225-233, 1995.

Fairfield, D. H.: Shape and location of the magnetopause, in Physics of the Magnetopause, (Eds) Song, Sonnerup and Thompsen, AGU Geophysical Monograph 90, 115-122, 1995.

Lucek, E. A., Dunlop, M. W., Horbury, T., Balogh, A., Cargill P., and the FGM team: Cluster magnetic field observations in the magnetosheath, Ann. Geophysicae, this issue, 2001.

Paschmann, G., Papamastorakis, I., Baumjohann, W., Sckopke, N., Carlson, C. W., Sonnerup, B. U. Ö., and Lühr, H.: ISEE observations of the magnetopause: Reconnection and the energy balance, J. Geophys. Res., 90, 12 111-12 120, 1985.

Paschmann, G., Papamastorakis, I., Baumjohann, W., Sckopke, N., Carlson, C. W., Sonnerup, B. U. Ö., and Lühr, H.: The Magnetopause for large magnetic shear: AMPTE/IRM observations, J. Geophys. Res., 91, 11 099-11 115, 1986.

Phan, T.-D., Paschmann, G., Baumjohann, W., Sckopke, N., and Lühr, H.: The magnetosheath region adjacent to the dayside magnetopause: AMPTE/IRM observations, J. Geophys. Res., 99, 121-141, 1994.

Phan, T.-D. and Paschmann, G.: The magnetosheath region adjacent to the dayside magnetopause, in: Physics of the Magnetopause, (Eds) Song, Sonnerup and Thompsen, AGU Geophysical Monograph 90, 115-122, 1995.

Russell, C. T.: The structure of the magnetopause, in: Physics of the Magnetopause, (Eds) Song, Sonnerup and Thompsen, AGU Geophysical Monograph 90, 81-98, 1995.

Russell, C. T. and Elphic, R. C.: Initial ISEE magnetometer results: Magnetopause observations, Space Sci. Rev., 22, 681-715, 1978.

Russell, C. T. and Elphic, R. C.: ISEE observations of flux transfer events at the dayside magnetopause, Geophys. Res. Lett., 6, 3336, 1979.

Shue, J. -H., Song, P., Russell, C. T., Steinberg, J. T., Chao, J. K., Zastenker, G., Vaisberg, O. L., Kokubun, S., Singer, H. J., and Detman, T. R.: Magnetopause location under extreme solar wind conditions, J. Geophys. Res., 103, 17, 691, 1998.

Shue, J. -H., Song, P., Russell, C. T., Chao, J. K., and Yang, Y. H.: Toward predicting the position of the magnetopause within Geosynchronous orbit, J. Geophys. Res., 103, 17, 691, 2000.

Sibeck, D. G., Lopez R. E., and Roelof, E. C.: Solar wind control of the magnetopause shape location and motion, J. Geophys. Res., 96, 5489, 1991.

Sonnerup, B. U. Ö. and Cahill, L. J.: Magnetopause structure and attitude from Explorer 12 observations, J. Geophys. Res., 72, 171-183, 1967.

Sonnerup, B. U. Ö. and Scheible, M.: 'Maximum and minimum variance analysis', in: Analysis Methods for Multispacecraft Data, ISSI Science Report, SR-001, Kluwer Academic Pub., 1998.

Tsyganenko N. A.: A magnetospheric field model with a warped tail current sheet, Planet. Space Sci., 37, 1, 5-20, 1989. 